\title{
Erratum to: Genome-wide association study of antibody level response to NDV and IBV in Jinghai yellow chicken based on SLAF-seq technology
}

\author{
Wenhao Wang • Tao Zhang • Genxi Zhang • Jinyu Wang • \\ Kunpeng Han • Yongjuan Wang • Yinwen Zhang
}

Published online: 5 March 2015

(C) Institute of Plant Genetics, Polish Academy of Sciences, Poznan 2015

\section{Erratum to: Journal of Applied Genetics}

\section{DOI 10.1007/s13353-014-0269-y}

Identified SNPs were named by the authors by taking 'rs', chromosome number and chromosomal basepair location.

Two SNPs were newly found and are not included in dbSNP database (The NCBI Short Genetic Variations database, build 141, http://www.ncbi.nlm.nih.gov/snp/):

rs16170823: chromosome 16 position 170823

rsZ2494710: chromosome Z position 2494710
Other six SNPs are included in the dbSNP database but they are named differently in the paper:

\begin{tabular}{ll}
\hline This paper & dbSNP \\
rs1211307701 & rs316966201 \\
rs1211307711 & rs312624692 \\
rs1218289310 & rs13716111 \\
rs420701988 & rs317837423 \\
rsZ2494661 & rs313388228 \\
rs149988433 & rs313017675 \\
\hline
\end{tabular}

The online version of the original article can be found at http://dx.doi.org/ 10.1007/s13353-014-0269-y.

W. Wang $\cdot$ T. Zhang $\cdot$ G. Zhang $\cdot$ J. Wang $(\bowtie) \cdot K$. Han College of Animal Science and Technology, Yangzhou University, Yangzhou, Jiangsu 225009, China

e-mail: jywang@yzu.edu.cn

W. Wang $\cdot$ T. Zhang $\cdot$ G. Zhang $\cdot$ J. Wang $\cdot$ K. Han Key Laboratory for Animal Genetics, Breeding, Reproduction and Molecular Design of Jiangsu Province, Yangzhou, Jiangsu 225009, China

Y. Wang

Jiangsu Jinghai Industry Poultry Group Co., Ltd.,

Nantong, Jiangsu 226103, China

Y. Zhang

Biomarker Technologies Corporation, Beijing, China 